\title{
XiUOS: an open-source ubiquitous operating system for industrial Internet of Things
}

\author{
Donggang $\mathrm{CAO}^{1,2^{*}}$, Dongliang $\mathrm{XUE}^{1,3}$, Zhiyi $\mathrm{MA}^{1,2}$ \& Hong $\mathrm{MEI}^{2}$ \\ ${ }^{1}$ Advanced Institute of Information Technology, Peking University, Hangzhou 311215, China; \\ ${ }^{2}$ Computer Science Department, Peking University, Beijing 100871, China; \\ ${ }^{3}$ Computer Science Department, Shanghai Jiao Tong University, Shanghai 200240, China
}

Received 11 May 2021/Accepted 11 June 2021/Published online 27 July 2021

Citation Cao D G, Xue D L, Ma Z Y, et al. XiUOS: an open-source ubiquitous operating system for industrial Internet of Things. Sci China Inf Sci, 2022, 65(1): 117101, https://doi.org/10.1007/s11432-021-3294-y

On May 4, 2021, a Chinese research team with members from AIIT (PKU Advanced Institute of Information Technology), PKU (Peking University), and SJTU (Shanghai Jiao Tong University), openly released the source code of XiUOS, a UOS (ubiquitous operating system) [1] for the industrial Internet of Things (IIoT), under the open-source license of Mulan PSL v2 on Trustie ${ }^{1)}$ and MulanOS ${ }^{2)}$. XiUOS is the first operating system developed from the UOS research. It is also one of the few OSs specially designed for supporting IIoT in intelligent manufacturing workshops. The UOS community hopes that the open-source release of XiUOS will help researchers and practitioners to carry out frontier UOS research in various real-world human-cyberphysical systems (HCPSs) [2].

Background: HCPS, UOS and IIoT. HCPS [2] is becoming an emerging trend in computing in recent years. HCPS is the extension of the cyber-physical system (CPS), where human beings are added to the CPS. HCPS has many new interesting features, e.g., everything getting digitalized, connection getting networked, system getting intelligent, and computation getting ubiquitous. These new features require the support of new OSs, whose capabilities are beyond current OSs. Therefore, professor Hong MEI at Peking University proposed to study UOS [1] to support HCPS application development, deployment, operation and management, and regarded UOS as an important OS development trend.

In the UOS technological systems, different HCPS application scenarios require different UOS instances. An intelligent manufacturing system is a typical HCPS in which the IIoT serves as a critical role for seamlessly interconnecting humans, cyber systems, and physical systems, especially those physical machines that were previously disconnected, to help workshops improving quality, increasing efficiency, reducing costs, and optimizing manufacturing process continuously. IIoT is highly complex and characterized by the in-depth integration of advanced information technology including IoT, AI, Edge computing, big data, with manufac- turing technology. To apply IIoT in an intelligent manufacturing factory/workshop, an IIoT platform or an operating system is needed to address the many issues like easy connectivity, convenient data acquisition, predictive maintenance, and on-demand control of physical machines.

Analyzing of current IIoT platforms. There are already many IIoT platforms in the market, most closed-source proprietary. These platforms can be broadly classified as industry agnostic or specific. Industry agnostic: mainly developed by software companies. There are Azure IoT, AWS IoT, Alibaba Cloud IoT, and many others focusing on data management on cloud, software AG Cumulocity strong in device management, IBM Watson IoT platform powerful in analytic capabilities, PTC ThingWorx competent in almost all aspects. Industry specific: mainly provided by leading manufacturing equipment vendors, focusing on creating value based on their own equipment. Typical platforms are Siemens Mindsphere, Bosch IoT Suite as a platform, ABB Ability, and Haier COSMOPlat. These industry-specific platforms usually cooperate with those industry-agnostic components to offer better IIoT services.

In China, a large number of factories are trying to upgrade to intelligent manufacturing. But many of them are facing tough difficulties, e.g., low digital level equipment that is hard to connect, limited budget, lacking information technology infrastructure, and complex requirements. These factories need a flexible IIoT platform that can be easily tailored for their needs, better open source if possible.

XiUOS development process. In March 2019, professor Hong MEI proposed to set up a team to develop a UOS for intelligent manufacturing scenarios, supporting IIoT applications. Some team members were from PKU having experience of developing the Internetware OS [3]. Other members were from SJTU having experience of developing softwaredefined in-memory computing system [4]. The government of Xiaoshan District, Hangzhou City, gave strong support for this research. In November 2019, the research was trans-

* Corresponding author (email: caodg@pku.edu.cn)

1) https://forgeplus.trustie.net.

2) https://mulanos.cn. 
Cao D G, et al. Sci China Inf Sci January 2022 Vol. 65 117101:2

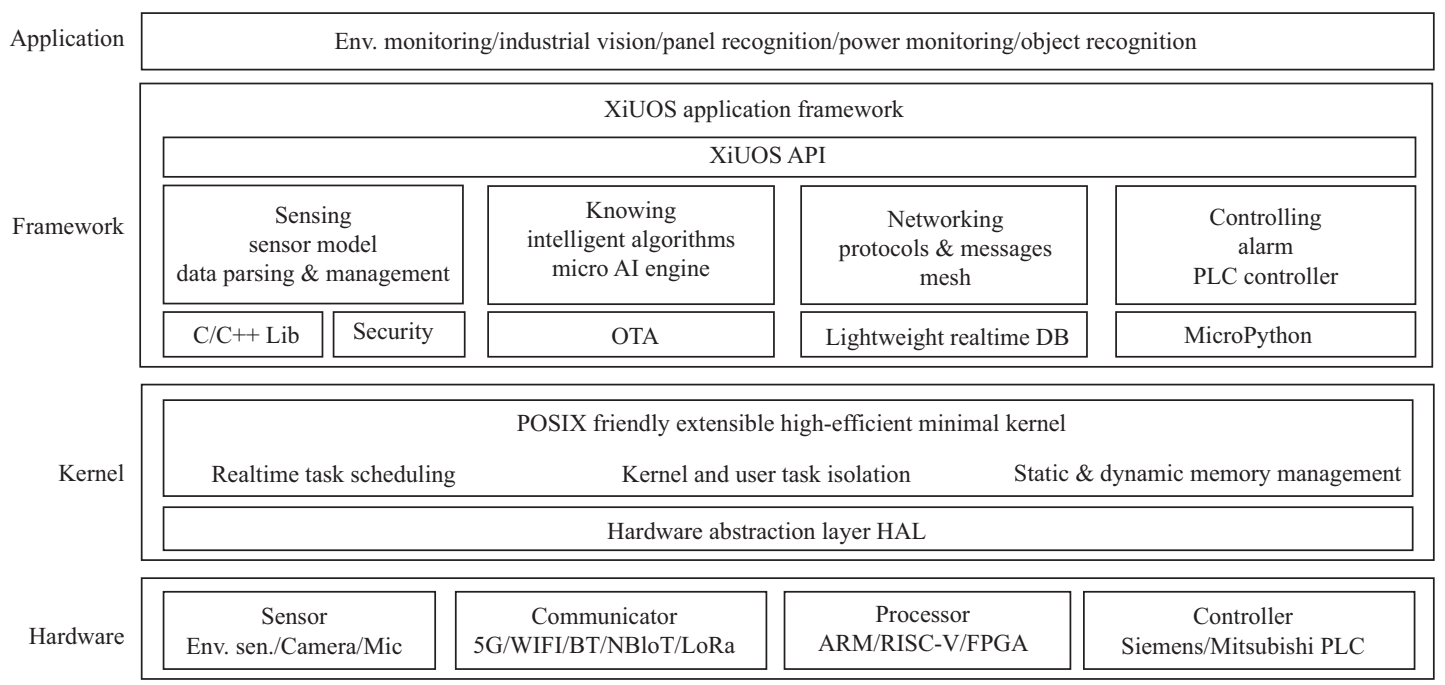

Figure 1 XiUOS architecture.

ferred to carry out in AIIT located at Xiaoshan. On May 4, 2021, version 1.0 of this UOS system was openly released as open-source, and the system was named XiUOS.

XiUOS design and features. XiUOS is designed to run on resource-limited IoT devices deployed in an industrial environment. XiUOS has a four-layered architecture as depicted in Figure 1, i.e., hardware layer, kernel layer, framework layer, and application layer.

In the hardware layer, XiUOS mainly supports the management of various sensors working in an industrial environment, sensing data of temperature, humidity, smoke, smell, noise, current, and voltage. It also supports cameras and microphones. About the communicator support, XiUOS provides drivers for $5 \mathrm{G} / 4 \mathrm{G}$, WIFI, NBIoT, LoRa, Ethernet, and ZigBee. XiUOS supports ARM and RISC-V processors. Now it can be run on 7 development boards and QEMU.

XiUOS has an extensible high-efficient minimal kernel. The kernel design considers the balance of abstraction, realtime performance, and efficiency, with features of realtime task scheduling, isolating kernel mode tasks from user mode, dynamic and static mixed memory management.

The IIoT application framework is the core part of XiUOS. Now the XiUOS version 1.0 has implemented basic sensing and networking functions, with preliminary intelligent analyzing and alarming control capabilities. The 2.0 version planned to release in December 2021 will implement a relatively complete IIoT application framework of "sensing, networking, knowing, and controlling", bringing about the benefits of convenient data acquisition, easy connectivity, predictive maintenance, and on-demand control of physical machines.

- Sensing. IIoT heavily relies on sensors to acquire data. However, it is difficult and error-prone for programmers to install sensor drivers, write sensing programs, and collect data. The XiUOS sensing framework provides a general physical-quantity oriented programming model, to simplify the sensor-related works such as data parsing, collecting, and management.

- Networking. The networking framework is designed to provide the commonly used network protocol stacks and message communication libraries such as MQTT (message queuing telemetry transport) to facilitate device connection and data exchange in a workshop environment. Wireless mesh network technology will be supported to ease network management.

- Knowing. The knowing framework aims at enabling AI in IoT device for realtime data analysis. AI algorithms speedup library and minimal AI engine are provided. The 1.0 version supports identifying instrument panel. The 2.0 will be able to identify voice, objects, humans, and defect patterns on resource-limited devices.

- Controlling. The controlling framework provides a PLC (programmable logic controller) controlling library, which can collect device data from PLCs of Siemens, Omron, and $\mathrm{B} \& \mathrm{R}$. Thus remote accurate controlling of machines becomes possible.

Some important features, including HAL (hardware abstraction layer), OTA (over-the-air), lightweight realtime DB, and MicroPython, are in development.

Applications. Two real cases have been implemented based on XiUOS, one is electric power monitoring for Qiantang River Electric Co., the other is workshop environment monitoring for Zhejiang Great Shengda Packaging Co.. Detailed information can be founded at the website ${ }^{3)}$.

Access methods. The XiUOS is published under the MulanPSL 2.0 open source license. Its source code is available at the websites ${ }^{4}$ )

\section{References}

1 Mei H, Guo Y. Toward ubiquitous operating systems: a software-defined perspective. Computer, 2018, 51: 50-56

2 Liu Z M, Wang J. Human-cyber-physical systems: concepts, challenges, and research opportunities. Front Inform Technol Electron Eng, 2020, 21: 1535-1553

3 Mei H, Guo Y. Operating systems for Internetware: challenges and future directions. In: Proceedings of the 38th International Conference on Distributed Computing Systems (ICDCS), 2018

4 Xue D L, Huang L P, Li C, et al. Dapper: an adaptive manager for large-capacity persistent memory. IEEE Trans Comput, 2019, 68: 1019-1034

3) http://xuos.io.

4) https://forgeplus.trustie.net/projects/xuos/xiuos.

5) https://github.com/xuos/xiuos. 